ISSN 0103-9954

\title{
ANÁLISE DA ESTRUTURA VERTICAL DE FLORESTAS ATRAVÉS DO DIAGRAMA h-M
}

\section{ANALYSIS OF THE VERTICAL STRUCTURE IN FORESTS THROUGH h-M DIAGRAM}

Carlos Roberto Sanquetta ${ }^{1}$

\begin{abstract}
RESUMO
Um método quantitativo de avaliação da estrutura vertical de florestas é desenvolvido. O método denominado diagrama h-M serve para o reconhecimento de estratos no povoamento como um todo e para populações especificas. Dados de uma floresta de "fir-hemlock" do Japão são usados para exemplificação da aplicação do método. Três estratos foram reconhecidos para o povoamento, o estrato I para as árvores emergentes superior a $30 \mathrm{~m}$ de altura, o II para as árvores de 5-30 de altura e o III para varas da regeneração, menores que $5 \mathrm{~m}$ de altura. Quatro tipos de estratificação de populações foram identificadas: a - espécies dominantes, com ampla estratificação; b - espécies pioneiras com apenas uma estrato; c - espécies não pioneiras (intermediárias) com dois estratos; d - espécies de sub-bosque com três estratos. As características de estratificação das diferentes populações foram relacionadas com seu comportamento ecológico. Concluiu-se que o diagrama h-M é uma ferramenta útil para a compreensão da estrutura de florestas.
\end{abstract}

Palavras-chave: estrutura; estratificação; "fir-hemlock"; Japão; populações.

\begin{abstract}
A quantitative method of analyzing the vertical structure of forests is proposed. The method was named h-M diagram and serves to distinguish stories in the forest as a whole or for specific populations. Data collected in a fir-hemlock forest in Japan were used to illustrate the usage of the diagram. The forest was composed of three stories: story I for emergent trees higher than $30 \mathrm{~m}$, II for trees of 5-30 m, and III for trees shorter than $5 \mathrm{~m}$. Four types of stratifications

1 Engenheiro Florestal, Ph.D., Professor do Departamento de Ciências Florestais, Universidade Federal do Paraná, Av. Pref. LothárioMeissner, 900,Jardim Botânico, CEP 80210-170, Curitiba (PR), Brasil.
\end{abstract}


Sanquetta, C.R.

were recognized for specific populations: a - dominant species with wide side distribution; $\mathrm{b}$ - pioneers with a single story; c - non-pioneer species (intermediate) with two stories; $d$ - understory species with three stories, the stratification of the populations was related to their ecological behavior. It was concluded that the h-M diagram is a helpful tool for understanding the structure of forests.

Keywords: structure; stratification; fir-hemlock; Japan; populations.

\section{INTRODUÇÃO}

Observando-se diversos povoamentos florestais, nota-se com frequencia a existencia de diferenciação vertical ou estratificação. Essa diferenciação é determinada sobretudo pela resposta ao decréscimo da disponibilidade de luz ao longo do perfil vertical da floresta (WHITTAKER, 1975).

A penetração de luz na floresta é uma função da disposição e da altura dos indivíduos ao longo do perfil vertical (KOHYAMA, 1989). Tal disposição é a causa maior da formação de grupos de plantas com hábitos similares, que ocupam mesmos nichos ecológicos e desempenham funções semelhantes (RICHARDS, 1979).

A avaliação da estrutura vertical é importante, uma vez que pode ser de grande valia para o manejo. A tarefa de reconhecer estratos de árvores na floresta é um dos principais elementos do estudo da estrutura vertical.

Vários métodos têm sido propostos para discriminar a presença de estratos na floresta (RICHARDS, 1979; OGAWA et al., 1963; MONSI \& SAEKI, 1953). Esses métodos são trabalhosos, parciais e aplicáveis a pequenas áreas e, as vezes destrutivos.

HOZUMI (1975) preocupou-se em desenvolver um método de estratificação que fosse menos subjetivo e prático quando propôs o diagrama M-w. Segundo NINOMIYA \& OGINO (1986), as vantagens do diagrama M-w são que ele independe do diâmetro mínimo de medição no inventário, é fácil de ser construído e a interpretação dos estratos é imediata.

O diagrama M-w é eficiente, porém um problema persiste, ou seja, estimativas de biomassa são necessárias. Por isso, NIMOMIYA et al. (1985), ,propuseram o uso do diâmetro transformado como variável representativa do peso. Todavia, é comum se observar, florestas naturais sobretudo, a baixa correlação do diâmetro com biomassa. Em tal a estratificação é problemática. 
Neste artigo o autor apresenta um novo método de análise da estratificação florestal. O método foi denominado diagrama h-M, e é uma modificação do método proposto por Hozumi, onde a variável altura é inserida como indicadora da estratificação.

\section{MATERIAL E MÉTODOS}

\section{Descrição da Área de Estudo}

Os dados utilizados no presente estudo foram coletados numa floresta natural de coníferas perenes "fir" e "hemlock" (Abies firma Sieb. \& Zucc. Tsuga sieboldii Carr.), composta também por um grande número de espécies folhosas caducifólias e perenes do sudeste do Japão.

A floresta situa-se na localidade de Komenono, a cerda de $25 \mathrm{Km}$ a oeste da cidade de Matsuyama, Província de Ehime, Shikoku (13254'E e $33^{\circ} 53^{\prime} \mathrm{N}$ ), a menor das quatro ilhas principais do Japão. A altitude varia de $760 \mathrm{~m}$ a $855 \mathrm{~m}$. A precipitação média anual é de $1.740 \mathrm{~mm}$, e as temperaturas mínima, média e máxima do ar registradas são $-6,2^{\circ} \mathrm{C}, 12,3^{\circ} \mathrm{C}$ e $35,5^{\circ} \mathrm{C}$, respectivamente (TOKUI \& SAKAUE, 1964; NINOMIYA et al., 1985).

Os solos são do tipo brunizen, BB, BC, BD, BD (d) de acordo com a classificação japonesa oficial (TSUJITA et al., 1986). A rocha mãe foi classificada como granito altamente intemperizado.

A vegetação pertence a aliança Hydrangeo-Abietetum firmae, classe Camellietea japonicae, conforme MIYAWAKI (1982). Ela cobre uma área transicional entre a zona temperada amena alta, dominada por folhosas sempre-verdes, e a zona temperada fria baixa, dominada por folhosas caducifólias (YAMANAKA, 1961).

\section{Descrição do Dados Utilizados}

Os dados deste trabalho foram coletados numa parcela permanente de 0,89 ha, subdividida em unidades de $10 \mathrm{~m} \times 10 \mathrm{~m}$, que foi instalada em dezembro de 1984. Todas as árvores acima de $4 \mathrm{~cm}$ em DAP (diâmetro a altura do peito; $1,30 \mathrm{~m}$ acima do nível do terreno) foram identificadas, rotuladas, mapeadas em escala e tiveram suas CAPs (circunferência a altura do peito) medidas. Um total de 2.637 árvores forma inventariadas, resultando numa área basal de $36,35 \mathrm{~m}^{2}$. Parte das alturas totais das árvores ocorrentes na 
Sanquetta, C.R.

parcela foram também medidas com aparelhos de princípio trigonométrico, sendo as demais alturas estimadas por equações hipsométricas desenvolvidas por grupos de espécies (NINOMIYA et al. 1985; SANQUETTA, 1994). Remedições anuais tem sido, sempre no mês de dezembro, mês de estagnação do crescimento (SANQUETTA et al., 1992).

Em setembro de 1991 treze sub-parcelas de $10 \mathrm{~m}$ x $10 \mathrm{~m}$ foram demarcadas para inventário dos indivíduos menores que $4 \mathrm{~cm}$ em DAP e mais de $20 \mathrm{~cm}$ de altura. Todos os indivíduos tiveram seus DAP's e alturas totas medidos. Um total de 2.500 indivíduos/ha foram registrados (SANQUETTA et al., 1992). As sub-parcelas também tem sido submetidas a remedições anuais.

Para este trabalho foram usados especificamente os dados coletados no ano de 1993, tanto das árvores de porte superior a $4 \mathrm{~cm}$ de DAP, bem como as varas de porte inferior a $4 \mathrm{~cm}$ em DAP e superior a $20 \mathrm{~cm}$ de altura. Detalhes dos dados e da floresta são reportados em SANQUETTA (1994).

\section{Análise da estrutura Dimensional pelo Diagrama h-M}

O método proposto por HOZUMI (1975), também conhecido como método M-N-Y, serve para reconhecimento do grupamento de árvores num espeço tridimensional. Este grupamento de árvores tem estreita relação com a estratificação vertical da floresta e com características especificas (NINOMIYA et al., 1985).

$\mathrm{O}$ método $\mathrm{M}-\mathrm{N}-\mathrm{Y}$, originado das palavras média (mean), número (number) e produção (yield), compõe-se das seguintes variáveis:

$\mathrm{f}(\mathrm{x})$ : função de distribuição de densidade de uma variável que caracteriza o tamanho individual das árvores $(\mathrm{x})$ numa certa unidade de área.

$\mathrm{N}(\mathrm{x}) \equiv \int \mathrm{x} \max \mathrm{f}(\mathrm{x}) \mathrm{dx}$ : número cumulativo de árvores de tamanho $\mathrm{x}$ a $\mathrm{X}{ }^{\text {max }}$ por unidade de área;

$\mathrm{Y}(\mathrm{X}) \equiv \int^{\mathrm{x} \max } \mathrm{x} \cdot \mathrm{f}(\mathrm{x}) \mathrm{dx}$ : produção cumulativa de árvores de tamanho $\mathrm{x}$ a $\mathrm{X}$ max por unidade de $\mathrm{n}$ área;

$\mathrm{M}(\mathrm{x})=\mathrm{N}(\mathrm{x}) / \mathrm{Y}(\mathrm{x})$ : valor médio de produção de uma população parcial de tamanho $\mathrm{x}$ a $\mathrm{x}{ }^{\max }$.

A relação de $\mathrm{M}$ e x em escala log-log foi originalmente chamada de diagrama M-w por HOZOMI (1975), uma vez que o autor usou o peso das árvores como variável x. NIMOMIYA et al. (1985) sugeriram que o valor DAP 5/2 fosse usado no lugar de w para simplificar o trabalho, em vista das 
dificuldades de se trabalhar com peso. O expoente $5 / 2$ foi justificado após inúmeros levantamentos de campo provarem que tal valor se repetia nas relações alométricas de peso sobre DAP.

Nesta pesquisa utilizou-se o método M-N-Y para análise da estrutura dimensional da floresta de "fir-hamlock" no Japão, porem com inovações. Em primeiro lugar, ao invés da variável peso, ou da sua correspondente do DAP elevado ao expoente $5 / 2$, utilizou-se a variável altura (h), uma vez que está é a variável que expressa mais propriamente a estratificação vertical da floresta. Para tanto tomou-se os dados das árvores que tiveram suas alturas medidas diretamente ou estimadas via relação hipsométrica.

Em segundo lugar, a posição de $\mathrm{M}$ e $\mathrm{h}$ foram invertidas em relação ao diagrama $\mathrm{M}-\mathrm{w}$, ou seja, na abcissa grafou-se $\mathrm{h}$ na ordenada $\mathrm{M}$. Isto facilita a interpretação do método de estratificação e a determinação dos mesmos.

Em terceiro lugar, não usou-se a escala log-log, como originalmente concebido, mas sim manteve-se a escala natural, porque a interpretação dos estratos na floresta se torna mais fácil. A esse novo método deu-se o nome de diagrama h-M, em correspondência ao diagrama $\mathrm{M}-\mathrm{w}$ originalmente propostos por HOZUMI (1975).

Além da análise da estrutura vertical do povoamento como um todo, usou-se o diagrama h-M para caracterizar a estrutura das principais espécies ocorrentes na floresta. O objetivo de caracterizar cada população separado foi procurar avaliar, de maneira simples, o comportamento e o hábito de tais espécies.

\section{RESULTADO E DISCUSSÃO}

\section{Estratificação Vertical do Povoamento como um todo}

O diagrama h-M do povoamento como um todo é apresentado na Figura 1. O diagrama reflete a estrutura vertical da floresta. Para compreender o significado prático do diagrama h-M basta observar a altura da floresta na ordenada em metros e o valor de $\mathrm{M}$, que corresponde ao valor médio cumulativo da variável h, que serve como variável auxiliar. é licito afirmar que a variável altura descreve melhor a estratificação vertical, porém se houver dificuldade de medição esta pode ser estimada por relações hipsométricas e utilizada no diagrama h-M sem problemas.

Os pontos que correspondem a cada árvore individualmente no 
povoamento, variam de zero até aproximadamente $35 \mathrm{~m}$ em h e M. Nota-se claramente que a partir do maior valor de $\mathrm{h}$ (árvore mais alta do povoamento) há uma queda gradual dos valores nos dois eixos, o qual é quebrada por uma queda abrupta em ambos. Nesses pontos, onde aparecem os picos indicados por flechas, a presença de estratos bem definidos é reconhecida. Caso tais picos não apareçam pode-se dizer que o povoamento não possui estratificação bem definida.

Com base no diagrama $\mathrm{h}-\mathrm{M}$, três estratos podem ser reconhecidos na floresta em estudo. O estrato I compõe-se de árvores acima de $30 \mathrm{~m}$ de altura, o estrato II de arvores de 5 a $30 \mathrm{~m}$, o estrato III é formado de árvores cujas alturas não ultrapassam $5 \mathrm{~m}$.

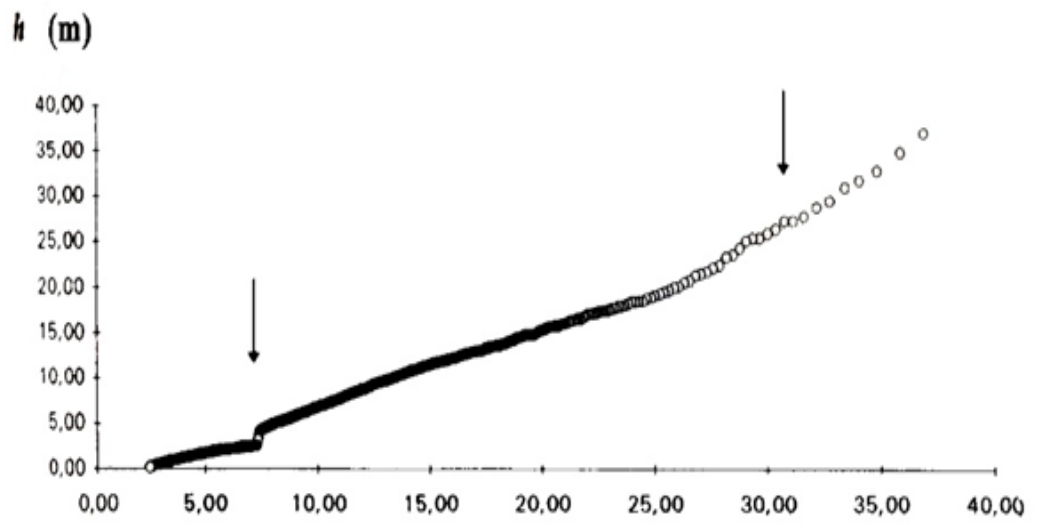

$M(\mathbf{m})$

FIGURA 1: Diagrama h-M para o povoamento como um todo de uma floresta de "fir-hemlock" no sudeste do Japão. Obs.: Flechas indicam divisão de estratos.

FIGURE 1: Diagram h-M for the settlement as a whole of a "fir-hemlock" forest in southeastern Japan. Note: Arrows indicate division of strata

Percebe-se ainda na Figura 1, pela densidade de pontos, que o estrato I é formado por árvores esporádicas, isto é, emergentes. O estrato II constitui o dossel principal formado por um bloco compacto de árvores. O estrato III 
também é denso e constitui o sub-bosque formado por varas de regeneração e espécies típicas da porção inferior da floresta.

\section{Estratificação Vertical das Populações}

Além da estratificação da floresta como um todo é possível fazer a análise da estratificação vertical de cada espécie, ou grupo de espécies, isto é, a estrutura dimensional dentro das populações. Através da avaliação da estrutura vertical em populações pode-se identificar o comportamento ecológico e o hábito de cada população. Esta analise prevê informações importantes para a compreensão das características de cada espécie, o que dá embasamento para entendimento das estratégias de regeneração natural, crescimento e sobrevivência. Esta avaliação pode ser feita da mesma forma que na casa da estratificação da floresta como um todo, lançando-se mão do diagrama h-M.

Vários tipos de estratificação de populações podem ser identificados, dependendo da floresta que se estuda. A Figura 2 resume os principais tipos de estratificação especiefica observadas na floresta em estudo. Tem-se basicamente quatro tipos:

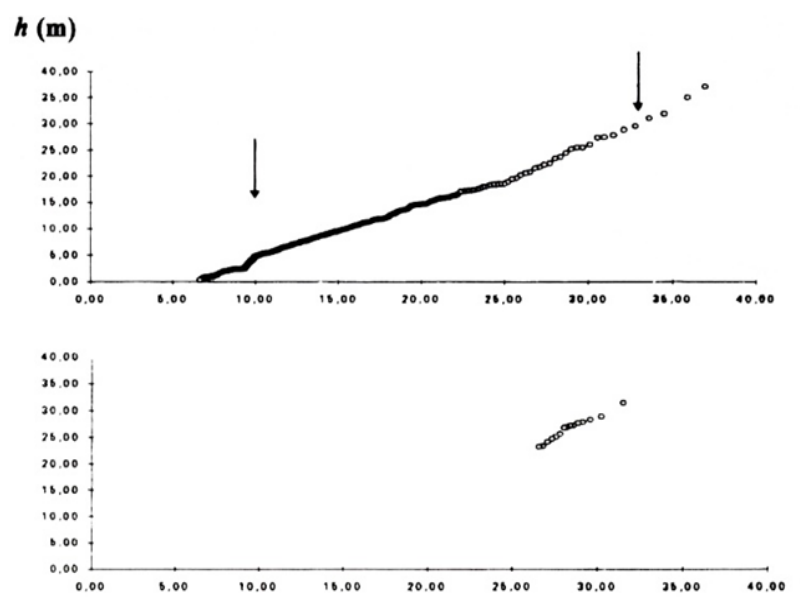

(a) 


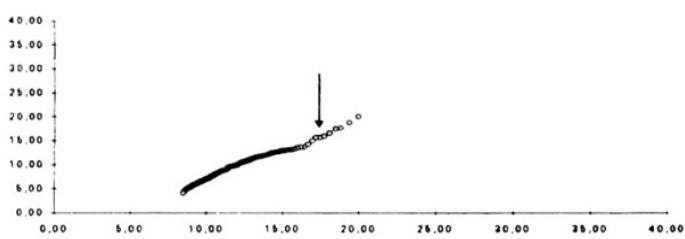

(c)

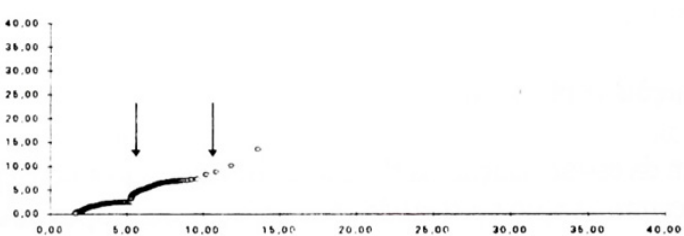

(d)

$M(\mathbf{m})$

FIGURA 2: Tipos de digrama h-M em populações de uma floresta de "fir-hemlock" no sudeste do Japão. (a) Coníferas; (b) folhosas caducas pioneiras; (c) folhosas caducas não pioneiras; (d) folhosas perenes não pioneiras. Obs.: Flechas indicam divisão de estratos.

FIGURE 2: Types of h-M diagram in populations of a fir-hemlock forest in southeastern Japan. (A) Conifer; (B) pioneer deciduous hardwoods; (C) non-pioneer deciduous hardwoods; (D) non-pioneering perennial hardwoods. Note: Arrows indicate division of strata.

-Tipo a: estratificação seguindo a estratificação da floresta como um todo, com ocorrência de ampla distribuição dimensional, isto é, presença desde indivíduos de grande porte (emergentes) até varas. Caracteriza espécies dominantes na floresta e com regeneração contínua sub sombra (clímax), como é a casa das coníferas na floresta em estudo.

-Tipo b: presença de apenas indivíduos de grande porte, se estratificação (um estrato) e ausência total de indivíduos de pequeno e médio portes. Caracteriza espécies pioneiras que se estabeleceram no passado, cresceram rapidamente para atingir o dossel superior, mas não regeneram sub sombra, apresentando déficit de mudas. Nesta floresta as espécies caducas pioneiras mostraram este tipo de distribuição dimensional.

-Tipo c: formação de dois estratos bem definidos, um com indivíduos que podem atingir porte até $20 \mathrm{~m}$ de altura e um com outros indivíduos de médio porte. Falta completa de indivíduos de pequeno porte também 
caracteriza espécies com dificuldade de regeneração sub sombra. Contudo a mais ampla distribuição de tamanhos indica que são espécies mais tolerantes que o tipo b. Na floresta em estudo, as espécies caducas não pioneiras exemplificam este tipo de distribuição dimensional.

-Tipo d: espécies de pequeno porte que não chegam a atingir o estrato I do povoamento e raramente atinge o estrato II. Tais espécies tem distribuição de tamanhos restrita, ou seja, são espécies confinadas no sub-bosque, regenerando continuamente sub sombra. Também podem ter estratificação interna, como é o caso das espécies perenes não pioneiras, que formam 3 estratos bem distintos de indivíduos.

O diagrama h-M é uma relação simples entre a altura e a altura cumulativa decrescente média. Apesar desta relação ser oriunda da distribuição de frequência de alturas (ou de diâmetros, quando usa-se relação hipsométrica) estas não guardam a mesma utilidade para definição de estratos na floresta. Nota-se, na Figura 3, que tanto através das distribuições hipsométricas como das diamétricas fica difícil a identificação de estratos no povoamento. Conforme mostra a Figura 3, isso independe do número e da amplitude das classes diamétricas. A razão de eficácia do diagrama h-M é o balanço de importância entre tamanho e frequência.

Existem, provavelmente, várias razões para a formação de estratos bem definidos numa floresta. Aspectos arquitetônicos das árvores de diferentes espécies e em vários estágios de desenvolvimento tem sido mencionados por HALLÉ et al. (1978). Todavia a hipótese mais aceita é aquela referente a diferenciação de crescimento causada pela existência de nichos relacionados as seções horizontais de disponibilidade de luz ao longo do perfil vertical da floresta (KOHYAMA, 1993). 


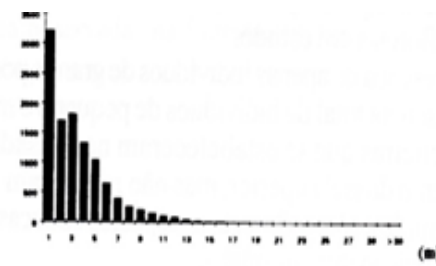

(a)

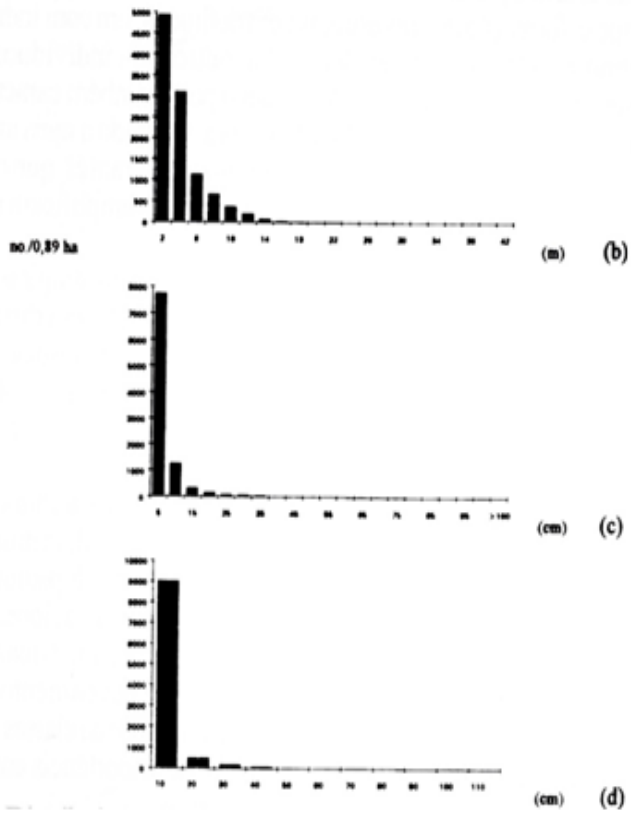

FIGURA 3: Distribuições de frequência de uma floresta de "fir-hemlock" no sudeste do Japão. (a) e (b) Distribuições de alturas totais; (c) e (d) Distribuições diamétricas.

FIGURE 3: Frequency distributions of a fir-hemlock forest in southeastern Japan. (A) and (b) Distributions of total heights; (C) and (d) Diametric distributions.

Neste trabalho, usando-se o diagrama h-M, identificou-se a existência de três estratos no povoamento de "fir-hemlock" no sudeste do Japão. Apesar de diferenças entre este povoamento e aqueles citados por RICHARDS 
(1975), notou-se uma concordância bastante significativa. O referido autor menciona que é comum o aparecimento de três estratos em florestas maduras. HOZUMI (1975) também chegou a essa conclusão, afirmando que qualquer povoamento florestal, por mais complexo que seja, geralmente é formado no máximo por quatro estratos.

O significado dos estratos neste povoamento foram caracterizados por SANQUETTA et al. (1994). O estrato de árvores mais altas é formado por coníferas esporádicas que não eram cortadas para fabrico de carvão antes da metade do século. Os membros do estrato imediatamente inferior forma uma copa densa de coníferas e folhosas caducas de praticamente mesma idade, os estratos mais baixos compõem-se, na sua maioria, de espécies perenes de estágios serais mais avançados, capazes de sobreviver sob sombra.

Outra utilidade prática do diagrama h-M é a possibilidade de reconhecimento de certas características ecológicas das espécies que compõem a floresta. Quatro tipos básicos forma reconhecidos: espécies clímax de grande porte (Figura 2-a), espécies pioneiras de grande porte (Figura 2-b), espécies secundárias ou intermediárias (Figura 2-c) e espécies de sub-bosque (Figura 2-d). Essas características especificas não foram originalmente identificadas por HOZUMI (1975), mas por NINOMIYA et al. (1985) e SANQUETTA et al. (1991).

\section{CONCLUSÕES}

Através deste estudo obteve-se as seguintes conclusões:

a) O diagrama h-M é um método eficiente e simples para o reconhecimento de estratos em povoamentos florestais;

b) A variável altura descreve melhor a estratificação vertical, porém se houver dificuldade de medição esta pode ser estimada por relações hipsométricas e utilizada no diagrama h-M sem problemas;

c) O número de estratos reconhecidos foi três: árvores emergentes, copa densa e sub-bosque, cada um formado por diferentes espécies;

d) A estratificação pode ser a nível de povoamento, com estratos compostos por diferentes espécies, assim como a nível de população;

e) A estratificação de populações revela certas características ecológicas das espécies florestais. 


\section{REFERÊNCIAS BIBLIOGRÁFICAS}

HALLÉ, F.; OLDEMAN, R.A.A.; TOMLINSON, P.B. Tropical trees and forests. Berlin-Heidelberg: Springer-Verlag, 1978. 441p.

HOZUMI, K. Studies on the frequency distribution of the weight of individual trees in a forest stand. V. The M-w diagram for various types of forest stands. Japanese Journal of Ecology, v.25, p.123-131, 1975.

KOHYAMA, T. Simulation of the structural development of warm-temperate rain forest stands. Annals of Botany, v.63, p.625-634, 1989.

KOHYAMA, T. Size-structured tree populations in gap-dynamic forest the forest architecture hypothesis for the strable coexistence of species. Journal of Ecology, v.81, p.131-143, 1993.

MIYAWAKI, A. Vegetation of Japan, Shikoku. Tóquio: Shibundo, 1983. 626p. (em japonês).

MONSI, M.; SAEKI, T. Uber den Lichtfaktor in den Pflanzengesellschaften und seine Bedeutung fur die Stoffproduktion. Japanese journal of Botany, v.14, p.22-52, 1953.

NINOMIYA, I.; HOTTA, M. (ed)., OGINO, K. Size structure by the M-w diagram. In: Diversity and dynamics of plant life in Sumatra, Sumatra Nature Study (Botany), Reports and Collection of Papers, Kyoto, 1986. p.17-27.

NINOMIYA, I. et al. diversity of species composition and stand structure in a natural secondary fir-hemlock forest. The Bulletin of Ehime University Forest, v.23, p.59-76, 1985 (em japonês, com resumo em inglês).

OGAWA, H.; SHINOZAKI, K.; HOZUMI, K. Quantitative analysis of plant community. In: ANNUAL MEETING OF THE BOTANICAL SOCIETY OF JAPAN, 26., 1963, Okayama. Annnual meeting... Okayama, 1963.

RICHARDS, P.W. The tropical rain forest. Cambridge: Cambridge University Press, 1979. 450p.

SANQUETTA, C.R. A model of natural regeneration of a fir-hemlock forest, southwestern Japan. Japão: UGAS, 1994. 136p. Tese (Doutorado em Agricultura) - Universidade de Ehime, 1994.

SANQUETTA, C.R.; NINOMIYA, I.; OGINO, K. Regeneration process in a fir-hemlock forest: analysis of sapling population. In: ANNUAL MEETING OF THE JANPANESE FORESTRY SOCIETY, 103., 1992, Tóquio. Transactions... Tóquio, 1992. p.357-359 (em japonês, entitulado em inglês).

SANQUETTA, C.R. et al. Age structural analysis of the natural 
regeneration process of a fir-hemlock secondary forest in southwest Japan. Journal of the Japanese Forestry Society, v.76, p.506-515, 1994.

SANQUETTA, C.R. et al. Dynamics during a 6-year period in a natural secondary fir-hemlock forest. The Bulletin of the Ehime University Forest, v.29, p.1-14, 1991.

TOKUI, O.; SAKAUE, M. Notes on the trees and shrubs in the Komenono University Forest. The Bulletin of the Ehime University Forest, v.2, p.2754, 1964. (em japonês, com resumo em inglês).

TSUJITA, A.; NIMOMIYA, I.; OGINO, K. Relation between the physical and chemical properties of forest soil and slope topography in a natural firhemlock forest of the Ehime University Forest. The Bulletin of the Ehime University Forest, v.24, p.59-76, 1986 ( em japonês, com resumo em inglês). WHITAKKER, R.H. Community and ecosystems, New Iork: MacMillan Publishing, 1975. 385p.

YAMANAKA, T. Abies firma and Tsuga sieboldii forest in Shikoku (Forest climaxes in Shikoku). Research Reports of Kochi University, Nature Series, v.10, p.1-14, 1961. 